\title{
Brodalumab to the Rescue: Efficacy and Safety of Brodalumab in Patients with Psoriasis and Prior Exposure or Inadequate Response to Biologics
}

\author{
Alan Menter · April Armstrong • Abby Van Voorhees • \\ Clive Liu $\cdot$ Abby Jacobson
}

Received: February 26, 2020 / Published online: June 12, 2020

(C) The Author(s) 2020

\section{ABSTRACT}

While biologic therapies for psoriasis are effective for many patients, some patients may lose response, have inadequate control of disease, or develop intolerance to certain biologic agents. It may therefore be beneficial for patients whose psoriasis fails to respond to one biologic to switch to a different biologic therapy, in particular one with a different mechanism of action. However, it remains unclear how prior biologic exposure or lack of response affects the efficacy and safety of subsequent biologics in patients with moderate-to-severe psoriasis.

Digital Features To view digital features for this article go to https://doi.org/10.6084/m9.figshare.12039699.

\section{A. Menter $(\square)$}

Baylor Scott \& White, Dallas, TX, USA

e-mail: amderm@gmail.com

A. Armstrong

University of Southern California, Los Angeles, CA, USA

A. Van Voorhees

Eastern Virginia Medical School, Norfolk, VA, USA

C. Liu

Bellevue Dermatology, Bellaire, TX, USA

A. Jacobson

Ortho Dermatologics (a division of Bausch Health

US, LLC), Bridgewater, NJ, USA
Brodalumab, a fully human anti-interleukin-17 receptor A monoclonal antibody, has previously been shown to be efficacious in treating moderate-to-severe psoriasis in three large phase 3 trials (AMAGINE-1, AMAGINE-2, and AMAGINE-3). In this review, we summarize the efficacy and safety of brodalumab in patients with moderate-to-severe psoriasis and a history of biologic exposure. Further, we describe improvements in skin clearance and quality of life measures as well as safety in patients who had inadequate response to ustekinumab and who were rescued with brodalumab therapy. Lastly, we discuss improvements in skin clearance following rescue with brodalumab in patients whose disease failed to respond to secukinumab and ixekizumab. The findings of our review suggest that brodalumab is a safe and efficacious treatment regardless of past biologic use or lack of response to prior biologic therapy.

Keywords: Interleukin-17 receptor; Loss of response; Psoriasis area and severity index; Static physician's global assessment 


\section{Key Summary Points}

Over the course of treatment, patients with psoriasis may lose response to biologic therapies, necessitating a switch to another therapy to achieve skin clearance.

This review demonstrates that long-term treatment with brodalumab, a fully human anti-interleukin-17 receptor A monoclonal antibody, produces skin clearance response rates that are similar between patients with or without a history of biologic exposure, with no difference in the safety profile of brodalumab between groups.

This review also demonstrates that treatment with brodalumab "rescued" skin clearance response in patients whose psoriasis did not respond to previous biologics, including ustekinumab, ixekizumab, or secukinumab.

\section{INTRODUCTION}

While biologic agents are generally effective for treating moderate-to-severe psoriasis, some patients have disease that "fails" to respond to biologic therapy. Primary failure occurs when a patient's disease does not initially respond to treatment. Secondary failure occurs when a patient's disease initially responds favorably to a medication but response is lost over time [1]. Importantly, loss of efficacy is the most common reason why patients discontinue biologic therapy [2, 3]. In a prospective observational cohort study, $23 \%$ of patients discontinued treatment (primarily because of loss of efficacy) within the first year of biologic therapy, and $47 \%$ discontinued after 3 years of treatment [4]. While discussed in generalities, the definition of treatment failure differs by drug or clinical study; prior studies have defined it as failure to achieve target static physician's global assessment (sPGA), psoriasis area and severity index (PASI), or investigator's global assessment scores [5-8].

Brodalumab is a fully human anti-interleukin-17 receptor A (IL-17RA) monoclonal antibody efficacious for the treatment of moderate-to-severe plaque psoriasis $[9,10]$. Although brodalumab is approved in Europe as a first-line biologic therapy for psoriasis [11], in the USA, it is approved only for use in adults who have experienced treatment failure with or loss of response to other systemic therapies [12]. Importantly, brodalumab has a unique mechanism of action among biologic therapies that targets the IL-17 pathway by blocking the IL17RA. By selectively and directly binding to this receptor, brodalumab blocks the induction of multiple inflammatory cytokines involved in psoriasis [13, 14].

To inform clinical treatment decisions, it is crucial to understand the efficacy and safety of brodalumab in patients with and without prior exposure to biologics and in patients whose psoriasis did not respond to previous biologic therapies. Thus, this review summarizes recent research studies that evaluate brodalumab, other biologic therapies for psoriasis, and patients with prior biologic exposure, as well as published data from brodalumab clinical studies. This article is based on previously conducted studies and does not contain any studies with human participants or animals performed by any of the authors.

\section{EFFICACY AND SAFETY OF BRODALUMAB IN PATIENTS WITH OR WITHOUT A HISTORY OF BIOLOGIC USE}

The efficacy and safety of brodalumab have been established in three large phase 3 trials: AMAGINE-1, AMAGINE-2, and AMAGINE-3 $[9,10]$. In these trials, prior biologic exposure was measured separately from prior biologic failure. Importantly, data from AMAGINE-2/3 show that a history of prior biologic exposure did not affect the efficacy of brodalumab over the initial 12-week induction phase and 
through 120 weeks of treatment $[15,16]$. Of 1236 patients in the AMAGINE-2/3 trials who received brodalumab $210 \mathrm{mg}$ every 2 weeks (Q2W) during the 12-week induction period, $27 \%(n=334)$ had prior biologic exposure; most of those patients $(n=296 ; 88.6 \%)$ had been treated with tumor necrosis factor- $\alpha$ (TNF- $\alpha)$ inhibitors. Overall, 73\% $(n=902)$ of patients were biologic naive. At week 12, the percentage of biologic-experienced and biologic-naive patients receiving brodalumab $210 \mathrm{mg}$ Q2W who achieved PASI 75 was $81.7 \%$ and $87.1 \%$, respectively. Among biologic-experienced patients, PASI 90 and PASI 100 responses were $63.8 \%$ and $39.5 \%$, respectively; among biologicnaive patients, PASI 90 and PASI 100 responses were $71.6 \%$ and $40.9 \%$, respectively. The incidence of treatment-emergent adverse events (TEAEs) at week 12 was similar in biologic-experienced and biologic-naive patients $(56.9 \%$ and $57.4 \%$, respectively). Common TEAEs were previously reported and included headache, upper respiratory tract infection, nasopharyngitis, and arthralgia [9].

In a separate long-term analysis of 3625 patients in AMAGINE-2/3 receiving $\geq 1$ dose of brodalumab, $27 \% \quad(n=963)$ were biologic experienced and 73\% $(n=2662)$ were biologic naive [16]. PASI 75 and PASI 100 response rates were maintained from week 52 through week 120 regardless of prior biologic usage (Fig. 1). At week $120,84.2 \%$ and $91.3 \%$ of biologic-experienced and biologic-naive patients, respectively, achieved PASI 75; 52.5\% and 59.8\%, respectively, achieved PASI 100. Health-related quality of life (HRQOL) scores and signs and symptoms of psoriasis were similar between groups at weeks 12 and 52. Across all years, rates of TEAEs were similar among biologic-experienced and biologic-naive patients.

In a separate analysis, brodalumab demonstrated long-term efficacy and safety regardless of prior response to TNF- $\alpha$ inhibitors [17] and for patients who received $\geq 1$ dose of brodalumab in the AMAGINE- $2 / 3$ studies, as observed analysis showed that $86.1 \%, 74.8 \%$, and $47.7 \%$ of patients with prior adalimumab failure and $90.8 \%, 63.8 \%$, and $40.4 \%$ of patients who had a prior response to adalimumab achieved PASI 75, 90, and 100, respectively, at week 52 [18]. Brodalumab also maintained long-term PASI response in patients who experienced treatment failure with 1,2 , or $\geq 3$ prior biologic therapies [19]. Therefore, these data indicate that brodalumab is well tolerated and efficacious in patients with or without a history of prior biologic use, including adalimumab.

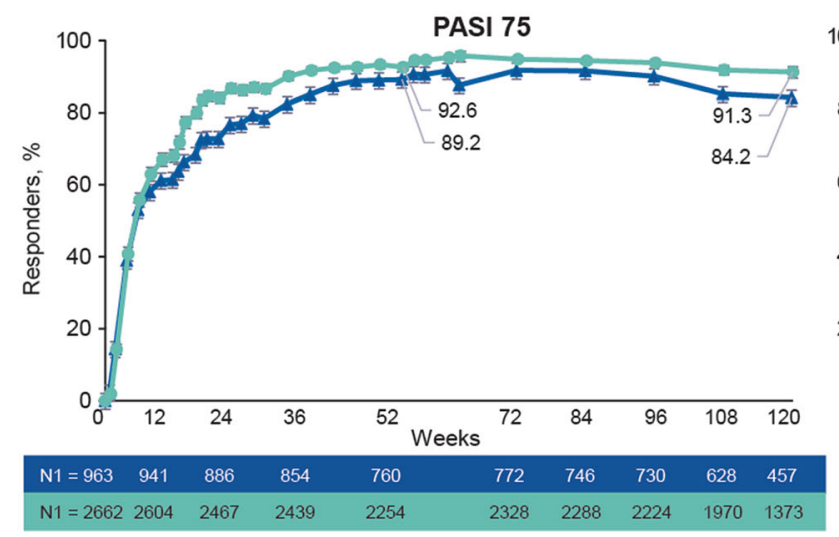

Fig. 1 PASI 75 and PASI 100 response rates in patients who received $\geq 1$ dose of brodalumab by prior biologic status at baseline. Error bars are 95\% confidence interval.

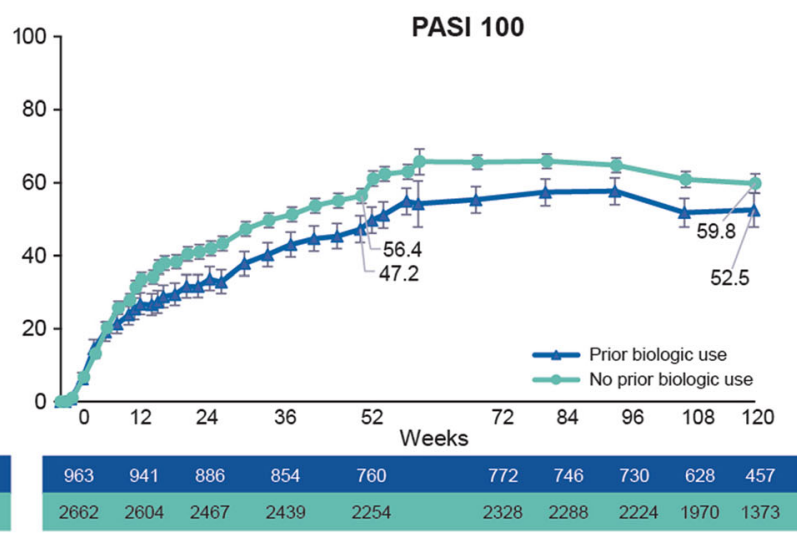

N1, number of patients with valid measurement at specified week; PASI 75 and 100, psoriasis area and severity index $75 \%$ and $100 \%$ improvement 


\section{EFFICACY AND SAFETY \\ OF BRODALUMAB IN PATIENTS WITH AN INADEQUATE RESPONSE TO USTEKINUMAB}

In the AMAGINE-2/3 trials, both of which included ustekinumab (a monoclonal antibody to the p40 subunit of IL-12 and IL-23) as an active comparator, $>50 \%$ of patients receiving brodalumab had complete clearance of psoriasis (i.e., PASI 100 within 1 year of treatment), compared with 30\% for ustekinumab [9]. A subgroup analysis of AMAGINE-2/3 evaluated patients with inadequate response to ustekinumab (defined as a single sPGA score $\geq 3$ or persistent sPGA score of 2 over $\geq 4$ weeks) [6]. These patients were either "rescued" with brodalumab $210 \mathrm{mg}$ Q2W starting at week 16 $(n=124)$ or continued on ustekinumab until week $52(n=149)$. Patients receiving ustekinumab who qualified for rescue after week 16 continued on ustekinumab until week 52 .

Of patients who experienced inadequate response to ustekinumab, those rescued with brodalumab $210 \mathrm{mg}$ Q2W tended to have higher rates of skin clearance at week 52 compared with patients who continued ustekinumab (Fig. 2). Patients who had been rescued with brodalumab $210 \mathrm{mg}$ Q2W tended to have higher skin clearance rates at week $52(72.6 \%$, $58.1 \%$, and $36.3 \%$ achieved PASI 75 , PASI 90 , and PASI 100, respectively) than patients who continued on ustekinumab $(61.7 \%, 25.5 \%$, and $5.4 \%$, respectively) $[6,20]$. Further, PASI 75 and PASI 90 response rates from week 12 to week 52 in patients rescued with brodalumab increased 3 -fold (24.2\% to $72.6 \%)$ and 11 -fold (4.8\% to $58.1 \%)$, respectively; PASI 100 response rates increased from $0 \%$ to $36.3 \%$.

Greater improvements in HRQOL and signs and symptoms of psoriasis were observed at week 52 in patients rescued with brodalumab relative to those who continued on ustekinumab after inadequate response [6]. Exposureadjusted rates of TEAEs in patients rescued with brodalumab were similar to those in patients who continued on ustekinumab [6]. Overall, brodalumab was efficacious and improved quality of life in patients with moderate-tosevere psoriasis who did not respond to ustekinumab.

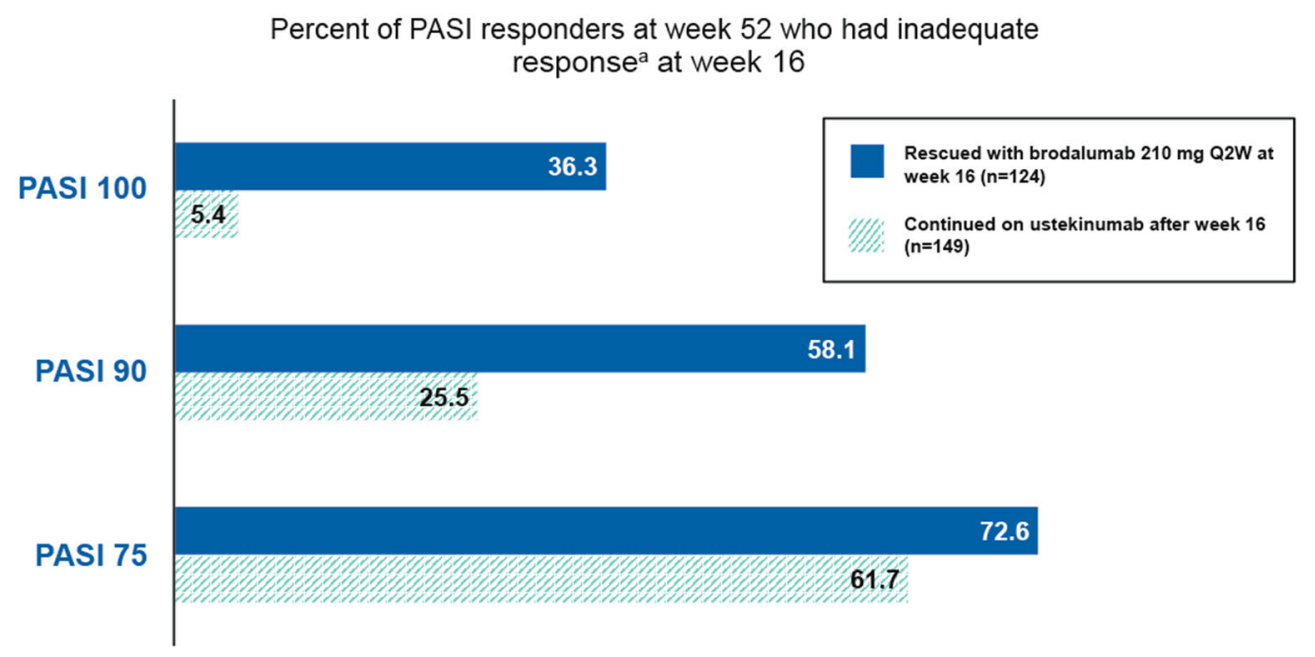

Fig. 2 Skin clearance response rates at week 52 in patients with inadequate response to ustekinumab after rescue with brodalumab $210 \mathrm{mg}$ Q2W. ${ }^{a}$ Inadequate response defined as single sPGA score $\geq 3$ or persistent sPGA score of 2 over $\geq 4$ weeks. PASI 75, 90, and 100, psoriasis area and severity index 75\%, 90\%, and 100\% improvement; Q2W, every 2 weeks; sPGA $0 / 1$, static physician's global assessment score of 0 or 1 


\section{EFFICACY OF BRODALUMAB IN PATIENTS \\ WITH AN INADEQUATE RESPONSE TO ANTI-IL-17A BIOLOGICS}

Further evidence of the efficacy and safety of brodalumab in patients who lost response to IL-17A biologics was provided in an open-label study of 39 patients with moderate-to-severe psoriasis [21]. There were 34 patients who completed all study visits through week 16 , with lack of efficacy as the most common reason for discontinuation. Failure was defined as treatment with either secukinumab or ixekizumab for $\geq 3$ months without achieving PASI 75 response or experiencing 50\% loss of original improvement. Forty-one percent of patients failed treatment with secukinumab, $49 \%$ failed treatment with ixekizumab, and $10 \%$ failed treatment with both. A substantial number of patients had severe psoriasis that did not respond to prior therapies, with an average of $\sim 2$ prior failed biologics. All patients received brodalumab $210 \mathrm{mg}$ Q2W up to week 16 .

Most patients who lost response to anti-IL-17A agents experienced improvement with brodalumab after 16 weeks of treatment (Fig. 3). At week 16, the percentages of patients who achieved PASI 75, PASI 90, and PASI 100 response rates were $76 \%, 50 \%$, and $32 \%$, respectively (observed data analysis). Six adverse events (none considered related to study drug) and no serious adverse events occurred during the study.

\section{DISCUSSION}

Patients with psoriasis often not uncommnly lose response to biologic agents and thus need to augment or switch therapies to achieve appropriate skin clearance. Here, we summarize the efficacy and safety of brodalumab in patients with moderate-to-severe psoriasis who had prior biologic exposure or experienced treatment failure.

Over $25 \%$ of patients in the AMAGINE-2/3 trials had prior exposure to biologic therapies, predominantly TNF- $\alpha$ inhibitors. PASI response rates in patients with or without previous biologic exposure were similar over the initial induction phase as well as over long-term treatment. Regardless of biologic history, patients receiving long-term brodalumab also reported similar quality of life (QOL) outcomes.

Additionally, brodalumab may be an efficacious and safe treatment option for patients with psoriasis whose disease has not responded appropriately to ustekinumab and anti-IL-17A agents. In patients whose psoriasis failed to respond to ustekinumab, skin clearance

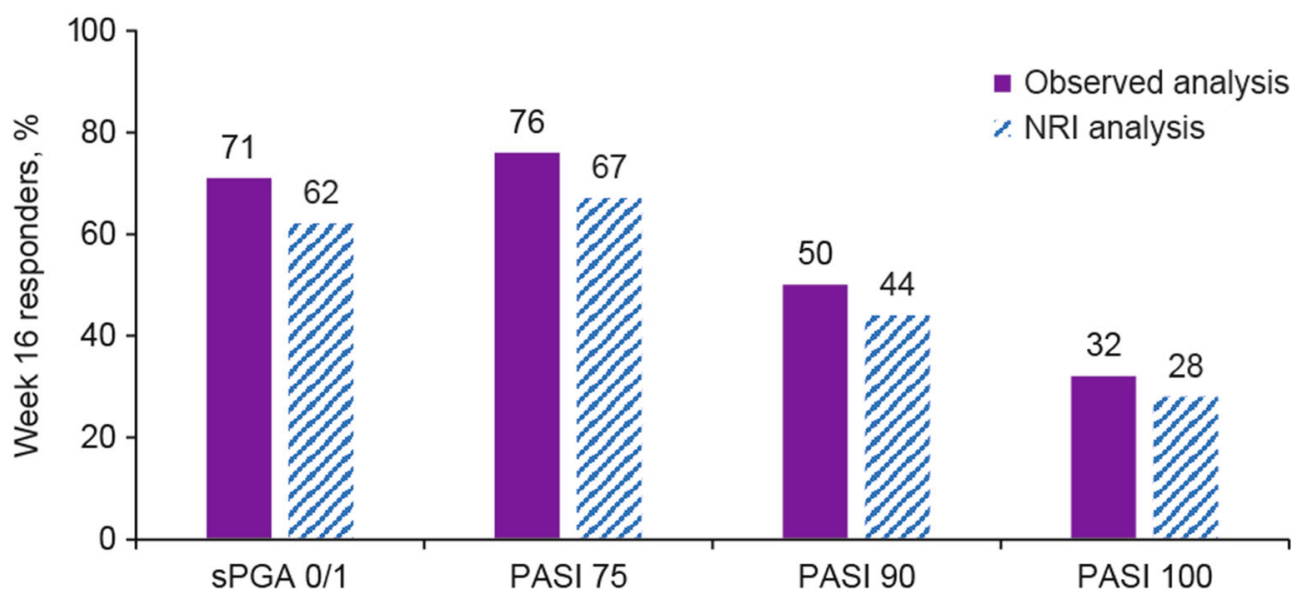

Fig. 3 Skin clearance response rates at week 16 in patients receiving brodalumab after previous inadequate response to an interleukin-17A inhibitor. NRI, nonresponder

imputation; PASI, psoriasis area and severity index; sPGA, static physician's global assessment 
response rates were increased through 36 weeks of brodalumab rescue treatment compared with individuals who had lost ustekinumab response but remained on the drug. In patients whose psoriasis failed to respond to the IL-17 inhibitors secukinumab or ixekizumab, brodalumab improved skin clearance after 16 weeks of treatment. In all studies, the safety profile of brodalumab was not affected by prior biologic exposure or in patients who had failed to respond.

\section{CONCLUSIONS}

Evidence presented herein demonstrates that brodalumab is an important option for patients regardless of prior biologic history and can improve skin clearance in patients who have lost response to prior biologic therapies. These findings may will be useful to clinicians seeking alternatives to ustekinumab or an anti-IL-17A biologic for efficacy-related reasons.

\section{ACKNOWLEDGEMENTS}

Funding. This analysis and the journal's Rapid Service Fee were supported by Ortho Dermatologics (a division of Bausch Health US, LLC).

Editorial Assistance. Editorial assistance was provided with support from Ortho Dermatologics (a division of Bausch Health US, LLC) under the direction of the authors by Deirdre Rodeberg, PhD, and David Boffa, ELS, at MedThink SciCom.

Authorship. All named authors meet the International Committee of Medical Journal Editors (ICMJE) criteria for authorship for this article, take responsibility for the integrity of the work as a whole, and have given their approval for this version to be published.

Disclosures. Alan Menter has received compensation from or served as an investigator, consultant, advisory board member, or speaker for Abbott Labs, AbbVie, Amgen, Boehringer Ingelheim, Celgene, Eli Lilly \& Co, Janssen Biotech, Leo, Merck \& Co, Novartis AG, Sun Pharma, and UCB. Alan Menter is a member of the journal's editorial board. April Armstrong has served as a research investigator, speaker, and/or consultant for AbbVie, BMS, Celgene, Dermavant, Dermira, Eli Lilly \& Co, Genentech, Genzyme, GSK, Janssen, Kyowa Hakko Kirin, Leo, Menlo Therapeutics, Modernizing Medicine, Novartis, Ortho Dermatologics, Pfizer, Regeneron, Sanofi, Science 37, Sun Pharma, UCB, and Valeant. Abby Van Voorhees has served as an investigator, consultant, or advisory board member for AbbVie, Allergan, Celgene, Dermira, Eli Lilly, Novartis, Ortho Dermatologics, and WebMD. Clive Liu has served on speaker bureaus and participated in research and advisory boards for AbbVie, Celgene, Janssen, Lilly, Novartis, Ortho Pharmaceuticals, Regeneron, Sanofi, and Sun Pharmaceuticals. Abby Jacobson is an employee of Ortho Dermatologics and holds stocks and/or stock options in Bausch Health.

Compliance with Ethics Guidelines. This article is based on previously conducted studies and does not contain any studies with human participants or animals performed by any of the authors.

Data Availability. The data that support the findings of this study are available from the corresponding author upon reasonable request.

Open Access. This article is licensed under a Creative Commons Attribution-NonCommercial 4.0 International License, which permits any non-commercial use, sharing, adaptation, distribution and reproduction in any medium or format, as long as you give appropriate credit to the original author(s) and the source, provide a link to the Creative Commons licence, and indicate if changes were made. The images or other third party material in this article are included in the article's Creative Commons licence, unless indicated otherwise in a credit line to the material. If material is not included in the article's Creative Commons licence and your intended use is not 
permitted by statutory regulation or exceeds the permitted use, you will need to obtain permission directly from the copyright holder. To view a copy of this licence, visit http:// creativecommons.org/licenses/by-nc/4.0/.

\section{REFERENCES}

1. Menter A, Strober BE, Kaplan DH, et al. Joint AADNPF guidelines of care for the management and treatment of psoriasis with biologics. J Am Acad Dermatol. 2019;80:1029-72.

2. Levin AA, Gottlieb AB, Au SC. A comparison of psoriasis drug failure rates and reasons for discontinuation in biologics vs conventional systemic therapies. J Drugs Dermatol. 2014;13:848-53.

3. Honda H, Umezawa Y, Kikuchi S, et al. Switching of biologics in psoriasis: reasons and results. J Dermatol. 2017;44:1015-9.

4. Warren RB, Smith CH, Yiu ZZN, et al. Differential drug survival of biologic therapies for the treatment of psoriasis: a prospective observational cohort study from the British Association of Dermatologists Biologic Interventions Register (BADBIR). J Investig Dermatol. 2015;135:2632-40.

5. Langley RG, Tsai TF, Flavin S, et al. Efficacy and safety of guselkumab in patients with psoriasis who have an inadequate response to ustekinumab: results of the randomized, double-blind, phase III NAVIGATE trial. Br J Dermatol. 2018;178:114-23.

6. Langley RG, Armstrong AW, Lebwohl MG, et al. Efficacy and safety of brodalumab in patients with psoriasis who had inadequate responses to ustekinumab: subgroup analysis of two randomized phase III trials. Br J Dermatol. 2019;180:306-14.

7. Reich K, Armstrong AW, Foley P, et al. Efficacy and safety of guselkumab, an anti-interleukin-23 monoclonal antibody, compared with adalimumab for the treatment of patients with moderate to severe psoriasis with randomized withdrawal and retreatment: results from the phase III, double-blind, placebo- and active comparator-controlled VOYAGE 2 trial. J Am Acad Dermatol. 2017;76:418-31.

8. Blauvelt A, Gooderham M, Iversen L, et al. Efficacy and safety of ixekizumab for the treatment of moderate-to-severe plaque psoriasis: Results through 108 weeks of a randomized, controlled phase 3 clinical trial (UNCOVER-3). J Am Acad Dermatol. 2017;77:855-62.
9. Lebwohl M, Strober B, Menter A, et al. Phase 3 studies comparing brodalumab with ustekinumab in psoriasis. N Engl J Med. 2015;373:1318-28.

10. Papp KA, Reich K, Paul C, et al. A prospective phase III, randomized, double-blind, placebo-controlled study of brodalumab in patients with moderate-tosevere plaque psoriasis. Br J Dermatol. 2016;175: 273-86.

11. Union Register of medicinal products for human use. European Commission. https://ec.europa.eu/ health/documents/community-register/html/ h1155.htm. Accessed 20 Mar 2020.

12. Siliq [package insert]. Bridgewater: Valeant Pharmaceuticals North America, LLC; 2017.

13. Papp KA, Leonardi C, Menter A, et al. Brodalumab, an anti-interleukin-17-receptor antibody for psoriasis. N Engl J Med. 2012;366:1181-9.

14. Russell CB, Rand H, Bigler J, et al. Gene expression profiles normalized in psoriatic skin by treatment with brodalumab, a human anti-IL-17 receptor monoclonal antibody. J Immunol. 2014;192: 3828-36.

15. Papp KA, Gordon KB, Langley RG, et al. Impact of previous biologic use on the efficacy and safety of brodalumab and ustekinumab in patients with moderate-to-severe plaque psoriasis: integrated analysis of the randomized controlled trials AMAGINE-2 and AMAGINE-3. Br J Dermatol. 2018;179: 320-8.

16. Green L, Menter A, Boh E, Israel R, Jacobson A. Brodalumab in patients with or without previous use of biologic agents: long-term findings from two phase 3 psoriasis studies. In: Poster presented at: 77th Annual Meeting of the American Academy of Dermatology; March 1-5, 2019; Washington, DC.

17. Strober B, Bhatia N, Keegan B, Armstrong A, Israel RJ, Jacobson A. Post hoc analysis of long-term efficacy and safety of brodalumab by prior anti-TNF biologic status. In: Poster presented at: Maui Derm for Dermatologists; January 26-30, 2019; Maui, HI.

18. Kerdel F, Lewitt GM, Drew S, Jacobson A. Long-term efficacy and safety in clinical trials of brodalumab by prior response to adalimumab. In: Poster presented at: 78th Annual Meeting of the American Academy of Dermatology; March 20-24, 2020; Denver, CO.

19. Lebwohl M, Green L, Bettencourt M, Fretzin S, Jacobson A. Long-term efficacy and safety of brodalumab in patients with psoriasis whose disease did not respond to prior biologics. In: Poster presented at: 39th Annual Fall Clinical Dermatology 